\title{
Diagnostic utility and limitations of glutamine synthetase and serum amyloid-associated protein immunohistochemistry in the distinction of focal nodular hyperplasia and inflammatory hepatocellular adenoma
}

\author{
Nancy M Joseph ${ }^{1}$, Linda D Ferrell ${ }^{1}$, Dhanpat Jain ${ }^{2}$, Michael S Torbenson ${ }^{3}$, Tsung-Teh Wu ${ }^{4}$, \\ Matthew M Yeh ${ }^{5}$ and Sanjay Kakar ${ }^{1,6}$ \\ ${ }^{1}$ Department of Anatomic Pathology, University of California, San Francisco, CA, USA; ${ }^{2}$ Department of \\ Pathology, Yale University, New Haven, CT, USA; ${ }^{3}$ Department of Pathology, Johns Hopkins, Baltimore, MD, \\ USA; ${ }^{4}$ Division of Anatomic Pathology, Mayo Clinic, Rochester, MN, USA; ${ }^{5}$ Department of Pathology, \\ University of Washington, Seattle, WA, USA and ${ }^{6}$ Department of Anatomic Pathology, University of \\ California and Veteran Affairs Medical Center, San Francisco, CA, USA
}

\begin{abstract}
Inflammatory hepatocellular adenoma can show overlapping histological features with focal nodular hyperplasia, including inflammation, fibrous stroma, and ductular reaction. Expression of serum amyloidassociated protein in inflammatory hepatocellular adenoma and map-like pattern of glutamine synthetase in focal nodular hyperplasia can be helpful in this distinction, but the pitfalls and limitations of these markers have not been established. Morphology and immunohistochemistry were analyzed in $\mathbf{5 4}$ inflammatory hepatocellular adenomas, 40 focal nodular hyperplasia, and 3 indeterminate lesions. Morphological analysis demonstrated that nodularity, fibrous stroma, dystrophic blood vessels, and ductular reaction were more common in focal nodular hyperplasia, while telangiectasia, hemorrhage, and steatosis were more common in inflammatory hepatocellular adenoma, but there was frequent overlap of morphological features. The majority of inflammatory hepatocellular adenomas demonstrated perivascular and/or patchy glutamine synthetase staining $(73.6 \%)$, while the remaining cases had diffuse $(7.5 \%)$, negative $(3.8 \%)$, or patchy pattern of staining $(15 \%)$ that showed subtle differences from the classic map-like staining pattern and was designated as pseudo map-like staining. Positive staining for serum amyloid-associated protein was seen in the majority of inflammatory hepatocellular adenomas (92.6\%) and in the minority of focal nodular hyperplasia (17.5\%). The glutamine synthetase staining pattern was map-like in $90 \%$ of focal nodular hyperplasia cases, with the remaining $10 \%$ of cases showing pseudo map-like staining. Three cases were labeled as indeterminate and showed focal nodular hyperplasia-like morphology but lacked map-like glutamine synthetase staining pattern; these cases demonstrated a patchy pseudo map-like glutamine synthetase pattern along with the expression of serum amyloid-associated protein. Our results highlight the diagnostic errors that can be caused by variant patterns of staining with glutamine synthetase and serum amyloid-associated protein in inflammatory hepatocellular adenoma and focal nodular hyperplasia.

Modern Pathology (2014) 27, 62-72; doi:10.1038/modpathol.2013.114; published online 28 June 2013
\end{abstract}

Keywords: c-reactive protein; focal nodular hyperplasia; glutamine synthetase; hepatocellular adenoma; serum amyloid associated protein

Correspondence: Dr S Kakar, MD, Department of Anatomic Pathology, University of California and VA Medical Center, 4150 Clement Street, San Francisco, CA 94121, USA.

E-mail: sanjay.kakar@University of CaliforniaSanFrancisco.edu Received 1 March 2013; revised 20 May 2013; accepted 21 May 2013; published online 28 June 2013
Focal nodular hyperplasia is a benign, non-neoplastic lesion that is thought to arise from a hyperplastic response to altered blood flow. ${ }^{1-3}$ Histologically, it is characterized by hyperplastic liver nodules that are separated by fibrous septa radiating from a central scar. The fibrous septa and central scar contain 
dystrophic thick-walled arteries with intimal thickening and poorly formed elastic lamina, a prominent ductular reaction and variable lymphocytic inflammation; interlobular bile ducts are absent. In the past, a subset of focal nodular hyperplasia with prominent sinusoidal dilatation was classified as telangiectatic focal nodular hyperplasia. $^{2,4,5}$ Based on imaging features, clonality results, and gene expression studies, these so-called 'telangiectatic focal nodular hyperplasia' are now classified as inflammatory (or telangiectatic) hepatocellular adenoma. ${ }^{6-10}$ This variant of adenoma is one of the four subtypes recognized in the recent World Health Organization classification: ${ }^{11}$ hepatocyte nuclear factor $1 \alpha$-inactivated, $\beta$-catenin-activated, inflammatory, and unclassified. The inflammatory (or telangiectatic) subtype of hepatocellular adenoma is characterized by sinusoidal dilatation, hemorrhage, inflammation, and ductular reaction. Due to overlapping morphological features, the distinction between focal nodular hyperplasia and inflammatory hepatocellular adenoma can be difficult on morphological grounds alone, especially when limited tissue is available in a liver biopsy.

The value of immunohistochemistry in this differential diagnosis has been recently explored. Glutamine synthetase is an enzyme involved in ammonia detoxification by combining it with glutamate to produce the amino-acid glutamine. In the normal liver, glutamine synthetase expression is limited to a narrow rim of hepatocytes around the central vein. This zonation is thought to be the result of $\beta$-catenin activation in the centrizonal hepatocytes, which may be the result of Wnt signaling from the central vein. ${ }^{12,13}$ In focal nodular hyperplasia, there is expansion of the $\beta$-catenin-activated centrizonal region, which leads to overexpression in a characteristic geographic map-like pattern. ${ }^{14-16}$ Diffuse cytoplasmic expression of glutamine synthetase is seen in a small subset of $\beta$-cateninactivated hepatocellular adenomas, but the map-like pattern typical of focal nodular hyperplasia is not observed in hepatocellular adenomas. On the other hand, inflammatory hepatocellular adenomas, but not focal nodular hyperplasia, show cytoplasmic staining with antibodies to acute phase reactants, including serum amyloid-associated protein and C-reactive protein. ${ }^{15,17,18}$ Inflammatory hepatocellular adenomas are frequently characterized by in-frame deletions of the interleukin-6 signaling transducer gp130 protein or mutations of activator of transcription 3 (STAT3). ${ }^{19,20}$ This leads to constitutive activation of the interleukin-6 pathway and expression of acute phase reactants, serum amyloid-associated protein and C-reactive protein.

We have observed that immunohistochemical expression patterns in focal nodular hyperplasia and inflammatory hepatocellular adenoma are diverse and do not always conform to the typical results described in the literature. This study examines the glutamine synthetase, serum amyloid-associated protein, and C-reactive protein staining patterns in focal nodular hyperplasia and inflammatory hepatocellular adenoma to determine the specificity of these markers and to identify variant staining patterns that can lead to diagnostic errors.

\section{Materials and methods}

\section{Case Selection and Histological Review}

The study group comprised 54 inflammatory hepatocellular adenoma (10 biopsy, 44 resection), 40 focal nodular hyperplasia (24 biopsy, 16 resection), and 3 indeterminate lesions (1 biopsy, 2 resection) obtained from the participating institutions. The study was approved by the Institutional Review Board. Hematoxylin and eosin (H\&E)-stained sections were reviewed to confirm the diagnosis and nine histological features were recorded: nodularity, fibrous bands, pseudo-portal tracts, naked arterioles in parenchyma, ductular reaction, lymphocytic inflammation, sinusoidal dilatation, hemorrhage, and steatosis. These features were scored on a scale of 0-3: 0-absent, 1-mild (one focus in the lesion), 2-moderate (at least 2 foci in the lesion, but involving less than half of the lesion), and 3 -marked (involving majority of the lesion). For hemorrhage and steatosis, a score of $\geq 1$ was considered positive, while scores of 2 or 3 were regarded positive for all other features.

\section{Immunohistochemistry}

Sections from formalin-fixed paraffin-embedded tissue were stained using an automated immunohistochemistry staining process (DAKO Autostainer Plus System or Leica Bond III System) at the University of California San Francisco Immunohistochemistry Lab. After incubation in primary antibodies (Table 1), detection for serum amyloidassociated protein and $\beta$-catenin was performed by incubating slides in a rabbit anti-mouse secondary followed by incubation in a horseradish peroxidaseconjugated anti-rabbit antibody, then developing with 3-3'-diaminobenzidine tetrahydrochloride chromogen. For glutamine synthetase, detection was performed by incubating in a horseradish peroxidase-conjugated secondary antibody then developed with diaminobenzidine chromogen.

Glutamine synthetase staining was classified as one of the following five patterns: (1) negative (no staining); (2) diffuse staining (moderate or strong cytoplasmic staining in $>50 \%$ of hepatocytes); (3) map-like (moderate or strong cytoplasmic staining in broad groups of interconnected hepatocytes with sparing of small islands of hepatocytes, typically adjacent to fibrous septa), further classified into focal $(<50 \%)$ and diffuse $(\geq 50 \%)$; (4) perivascular and/or patchy staining (predominantly perivascular staining or perivascular staining accompanied by 
Table 1 Antibodies used for immunohistochemistry

\begin{tabular}{|c|c|c|c|c|}
\hline Antibody & Clone & Source & Dilution & Antigen retrieval \\
\hline Glutamine synthetase & GS-6 & MAB302, Millipore, Temecula, CA, USA & $1: 250$ & \multirow{2}{*}{$\begin{array}{l}\text { pH } 6.0 \text { citrate } \\
\text { buffer } \\
\text { pH } 6.0 \text { citrate } \\
\text { buffer }\end{array}$} \\
\hline $\begin{array}{l}\text { Serum amyloid-associated } \\
\text { protein }\end{array}$ & Mc1 & $\begin{array}{l}\text { M0759, DAKO Cytomation, Carpinteria, CA, } \\
\text { USA }\end{array}$ & $1: 500$ & \\
\hline$\beta$-Catenin & Clone 14 & 610154, BD Biosciences, San Jose, CA, USA & $1: 400$ & \multirow{2}{*}{$\begin{array}{l}\text { pH } 6.0 \text { citrate } \\
\text { buffer } \\
\text { pH } 6.0 \text { citrate } \\
\text { buffer }\end{array}$} \\
\hline C-reactive protein & $\begin{array}{l}\text { Rabbit monoclonal } \\
\text { Y284 }\end{array}$ & Ab32412, Abcam, Cambridge, MA, USA & 1:1500 & \\
\hline
\end{tabular}

staining of variable intensity in clusters of hepatocytes beyond perivascular region); and (5) pseudo map-like (similar to pattern (4), with the staining in clusters of hepatocytes beyond perivascular region showing interconnected areas, resembling map-like pattern). Pseudo map-like staining differed from map-like staining in three respects: (1) the positive interconnected bands of hepatocytes were narrow and inconspicuous, (2) intensity of staining was variable, typically mild to moderate, and faded in intensity away from perivascular areas and (3) focal and confined to periphery.

Serum amyloid-associated protein and C-reactive protein staining was scored on a scale of 0-3 for intensity, with 0 indicating absence of staining and 3 indicating strong staining. The positive cases were further classified as focal $(<50 \%)$ and diffuse $(\geq 50 \%)$. $\beta$-Catenin staining was classified as either membranous or nuclear staining. Staining patterns for all three antibodies were also recorded in adjacent uninvolved liver when present.

\section{Statistical Analysis}

The differences between clinical features, histological features, glutamine synthetase staining, and serum amyloid-associated protein staining in focal nodular hyperplasia and inflammatory hepatocellular adenoma were analyzed using the Fisher's exact test and the chi-square test.

\section{Results}

\section{Clinical and Histological Features}

Age and gender data were available for 43 inflammatory hepatocellular adenomas and 35 focal nodular hyperplasia. Of the 43 inflammatory hepatocellular adenoma cases, $37(86 \%)$ were female and had a mean age of 40 (range 3-65) years. Most patients with focal nodular hyperplasia were also women $(31 / 35,89 \%)$ with a mean age of 43 (range 24-73) years.

The comparison of histological features is summarized in Table 2. Nodular architecture, fibrous bands and ductular reaction were significantly
Table 2 Histological features of focal nodular hyperplasia (FNH) and inflammatory hepatocellular adenoma (IHA)

\begin{tabular}{|c|c|c|c|c|}
\hline & $\begin{array}{c}F N H \\
(\mathrm{n}=40)\end{array}$ & $\begin{array}{c}I H A \\
(\mathrm{n}=54)\end{array}$ & $\begin{array}{c}\mathrm{P}- \\
\text { value* }\end{array}$ & $\begin{array}{l}\text { Indeterminate } \\
\quad(\mathrm{n}=3)\end{array}$ \\
\hline $\begin{array}{l}\text { Nodular } \\
\text { architecture }\end{array}$ & $34 / 40$ (85) & $4 / 54(7.4)$ & $<0.001$ & 1/3 (33) \\
\hline Fibrous bands & $36 / 40$ (90) & $14 / 54(26)$ & $<0.001$ & 1/3 (33) \\
\hline Naked arterioles & $8 / 36(22)$ & $30 / 50(60)$ & 0.001 & $1 / 3(33)$ \\
\hline $\begin{array}{l}\text { Ductular } \\
\text { reaction }\end{array}$ & $33 / 40(83)$ & $23 / 54(43)$ & $<0.001$ & $1 / 3(33)$ \\
\hline $\begin{array}{l}\text { Pseudo-portal } \\
\text { tracts }\end{array}$ & $8 / 36(22)$ & $36 / 50(72)$ & $<0.001$ & 0 \\
\hline $\begin{array}{l}\text { Lymphocytic } \\
\text { inflammation }\end{array}$ & $19 / 40(48)$ & $32 / 53(60)$ & 0.08 & $1 / 3(33)$ \\
\hline $\begin{array}{l}\text { Sinusoidal } \\
\text { dilatation }\end{array}$ & 7/39 (18) & 44/53 (83) & $<0.001$ & $1 / 3(33)$ \\
\hline Hemorrhage & $2 / 39(5)$ & $35 / 50(70)$ & $<0.001$ & 0 \\
\hline Steatosis & 8/39 (21) & $31 / 54(57)$ & $<0.001$ & 0 \\
\hline
\end{tabular}

* $P$-values reflect comparison between focal nodular hyperplasia (FNH) and inflammatory hepatocellular adenoma (IHA); numbers in parentheses reflect percentages.

more common in focal nodular hyperplasia, while inflammation, sinusoidal dilatation, hemorrhage, naked arterioles, pseudo-portal tracts, and steatosis were significantly more common in inflammatory hepatocellular adenoma. As opposed to fibrous septa, pseudo-portal tracts showed a normal-sized arteriole, lacked a prominent ductular reaction, and appeared similar to a normal portal tract, except that there was no interlobular bile duct.

\section{Glutamine Synthetase and Serum Amyloid-Associated Protein Staining Patterns in Focal Nodular Hyperplasia}

Map-like glutamine synthetase staining pattern was observed in $36(90 \%)$ focal nodular hyperplasia cases (Table 3, Figure 1); the staining was focal in 7 $(17 \%)$ and diffuse in $29(73 \%)$ cases. In $4(10 \%)$ focal nodular hyperplasia cases, the typical map-like pattern was not seen. These cases showed a pseudo map-like glutamine synthetase staining throughout the lesion. All seven cases of focal 
Table 3 Glutamine synthetase (GS) immunohistochemical staining patterns in focal nodular hyperplasia (FNH) and inflammatory hepatocellular adenoma (IHA)

\begin{tabular}{|c|c|c|c|c|c|}
\hline & GS, map-like & GS, patchy and/or perivascular & GS, pseudo map-like & $G S$, diffuse & GS, negative \\
\hline FNH all cases $(n=40)$ & $36 / 40(90)$ & 0 & $4 / 40(10)$ & 0 & 0 \\
\hline FNH biopsy $(n=24)$ & $20 / 24(83)$ & 0 & $4 / 24(17)$ & 0 & 0 \\
\hline FNH resection $(n=16)$ & $16 / 16(100)$ & 0 & 0 & 0 & 0 \\
\hline IHA all cases $(n=53)$ & 0 & $39 / 53(74)$ & 8/53 (15) & $4 / 53(8)$ & $2 / 53(4)$ \\
\hline IHA biopsy $(n=9)$ & 0 & $7 / 9(78)$ & $1 / 9(11)$ & $1 / 9(11)$ & 0 \\
\hline IHA resection $(n=44)$ & 0 & $32 / 44(73)$ & $7 / 44(16)$ & $3 / 44(7)$ & $2 / 44(5)$ \\
\hline$P$-value & $<0.001$ & $<0.001$ & 0.47 & 0.07 & 0.22 \\
\hline Indeterminate lesions $(n=3)$ & 0 & 0 & $3 / 3(100)$ & 0 & 0 \\
\hline
\end{tabular}

$P$-values reflect comparison between focal nodular hyperplasia (FNH) and inflammatory hepatocellular adenoma (IHA); numbers in parentheses reflect percentages.
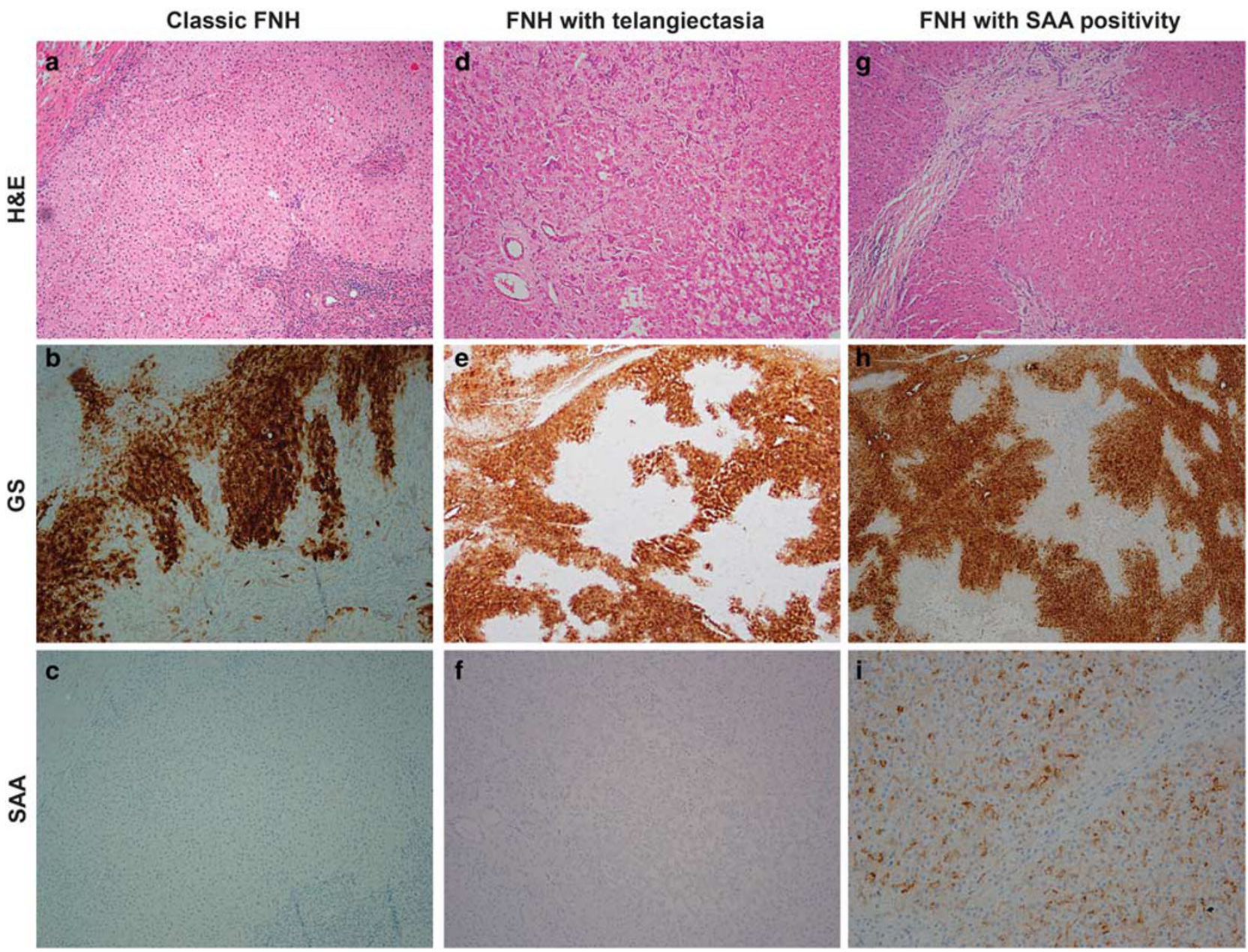

Figure 1 Glutamine synthetase and serum amyloid-associated protein staining patterns in focal nodular hyperplasia. (a-c) H\&E-stained image showing ductular reaction and fibrous septa (a), map-like glutamine synthetase staining (b), and negative results with serum amyloid-associated protein (c). (d-f) H\&E-stained image showing sinusoidal dilatation mimicking inflammatory hepatocellular adenoma (d), map-like glutamine synthetase staining (e), and negative results with serum amyloid-associated protein (f). (g-i) H\&E-stained image showing ductular reaction and fibrous septa (g), map-like glutamine synthetase staining (h), and positive cytoplasmic staining with serum amyloid-associated protein (i).

and all four cases of pseudo map-like glutamine synthetase staining were biopsies, while typical map-like staining was seen in all resection cases. Serum amyloid-associated protein was negative in
$33(83 \%)$ focal nodular hyperplasia cases (Table 4, Figure 1). Of the seven cases that were serum amyloid-associated protein positive, two were patchy and five were diffuse. 
Table 4 Serum amyloid associated (SAA) protein immunohistochemistry in focal nodular hyperplasia (FNH) and inflammatory hepatocellular adenoma (IHA)

\begin{tabular}{lcccc}
\hline & SAA, positive (any intensity) & SAA, negative (0) & SAA, positive (2 and 3) & SAA, negative (0 and 1) \\
\hline FNH all cases $(n=40)$ & $7 / 40(17)$ & $33 / 40(83)$ & $4 / 40(10)$ & $2 / 24(8)$ \\
FNH biopsy $(n=24)$ & $4 / 24(17)$ & $20 / 24(83)$ & $2 / 16(12)$ & $36 / 40(90)$ \\
FNH resection $(n=16)$ & $3 / 16(19)$ & $13 / 16(81)$ & & $22 / 24(92)$ \\
& & & $46 / 54(85)$ & $14 / 16(12)$ \\
IHA all cases $(n=54)$ & $50 / 54(93)$ & 0 & $9 / 10(90)$ & $8 / 54(15)$ \\
IHA biopsy $(n=10)$ & $10 / 10(100)$ & $4 / 44(9)$ & $37 / 44(87)$ & $7 / 10(10)$ \\
IHA resection $(n=44)$ & $40 / 44(100)$ & $<0.001$ & $<0.001$ & $<0.001$ \\
P-value & $<0.001$ & 0 & $3 / 3(100)$ & 0 \\
Indeterminate lesions $(n=3)$ & $3 / 3(100)$ & &
\end{tabular}

$P$-values reflect comparison between focal nodular hyperplasia (FNH) and inflammatory hepatocellular adenoma (IHA); numbers in parentheses reflect percentages.

\section{Glutamine Synthetase and Serum Amyloid-Associated Protein Staining Patterns in Inflammatory Hepatocellular Adenoma}

The following four glutamine synthetase staining patterns were observed in inflammatory hepatocellular adenoma (Table 3, Figures 2 and 3):

(1) Negative: 2 (4\%);

(2) Perivascular and/or patchy staining: 39 (74\%);

(3) Diffuse: 4 (8\%); nuclear $\beta$-catenin staining was seen in three of these cases; and

(4) Pseudo map-like: 8 (15\%). This pattern was observed focally at the periphery of the lesion. The remaining lesion showed perivascular and/or patchy staining. Typical map-like staining was not seen in any case. Serum amyloid-associated protein was positive in 50 (93\%, Table 4, Figure 2) cases, with focal staining in 6 and diffuse staining in 44 cases.

\section{Glutamine Synthetase and Serum Amyloid-Associated Protein Staining Patterns in Adjacent Uninvolved Liver}

Adjacent uninvolved liver was available for evaluation in 23 cases of inflammatory hepatocellular adenoma and 9 cases of focal nodular hyperplasia. Glutamine synthetase staining was seen in the perivenular region in the adjacent uninvolved liver in all the cases. ${ }^{15}$ Serum amyloid-associated protein staining was observed in the adjacent liver in 14 of 23 cases $(61 \%)$ of inflammatory hepatocellular adenoma and 5 of 9 cases (56\%) of focal nodular hyperplasia (Figure 4). In all 14 positive inflammatory hepatocellular adenoma cases, serum amyloid-associated protein staining was less intense in the adjacent normal liver compared with the inflammatory hepatocellular adenoma. In 3 of the 5 focal nodular hyperplasia cases that showed serum amyloid-associated protein staining in the adjacent normal liver, there was no serum amyloid-associated protein staining in the focal nodular hyperplasia. In two of the five cases, serum amyloid-associated protein staining was seen at the same level of intensity in both the focal nodular hyperplasia and adjacent normal liver.
C-Reactive Protein Staining Results in Focal Nodular Hyperplasia and Inflammatory Hepatocellular Adenoma

C-reactive protein staining was done in 27 focal nodular hyperplasia (12 biopsy, 15 resection) and 20 inflammatory hepatocellular adenoma (2 biopsy, 18 resection) cases. All inflammatory hepatocellular adenoma cases $(100 \%)$ demonstrated diffuse staining for C-reactive protein. Among focal nodular hyperplasia cases, C-reactive protein was positive in $78 \%$ of cases, with diffuse staining in $15 \%$ of cases.

\section{Morphology and Immunostaining Patterns in Indeterminate Lesions}

Based on the morphology and immunohistochemical features, three cases could not be classified as focal nodular hyperplasia or inflammatory hepatocellular adenoma and were labeled as indeterminate (Figure 5).

(1) Case 1: Morphology resembled focal nodular hyperplasia (nodularity, fibrous bands, ductular reaction, and inflammation), serum amyloid-associated protein positive, and glutamine synthetase pseudo map-like.

(2) Case 2: Overlapping morphological features (prominent dystrophic arterioles, sinusoidal dilatation, and ductular reaction), serum amyloid-associated protein positive, and glutamine synthetase pseudo map-like staining.

(3) Case 3: Morphology resembled focal nodular hyperplasia (slight nodularity, fibrous bands, ductular reaction), serum amyloid-associated protein positive, and glutamine synthetase pseudo map-like.

\section{Discussion}

Focal nodular hyperplasia is considered a nonneoplastic lesion and does not need surgical resection even if it is large, unless the patient is symptomatic or the location of the lesion compromises liver function. ${ }^{6}$ On the other hand, inflammatory 

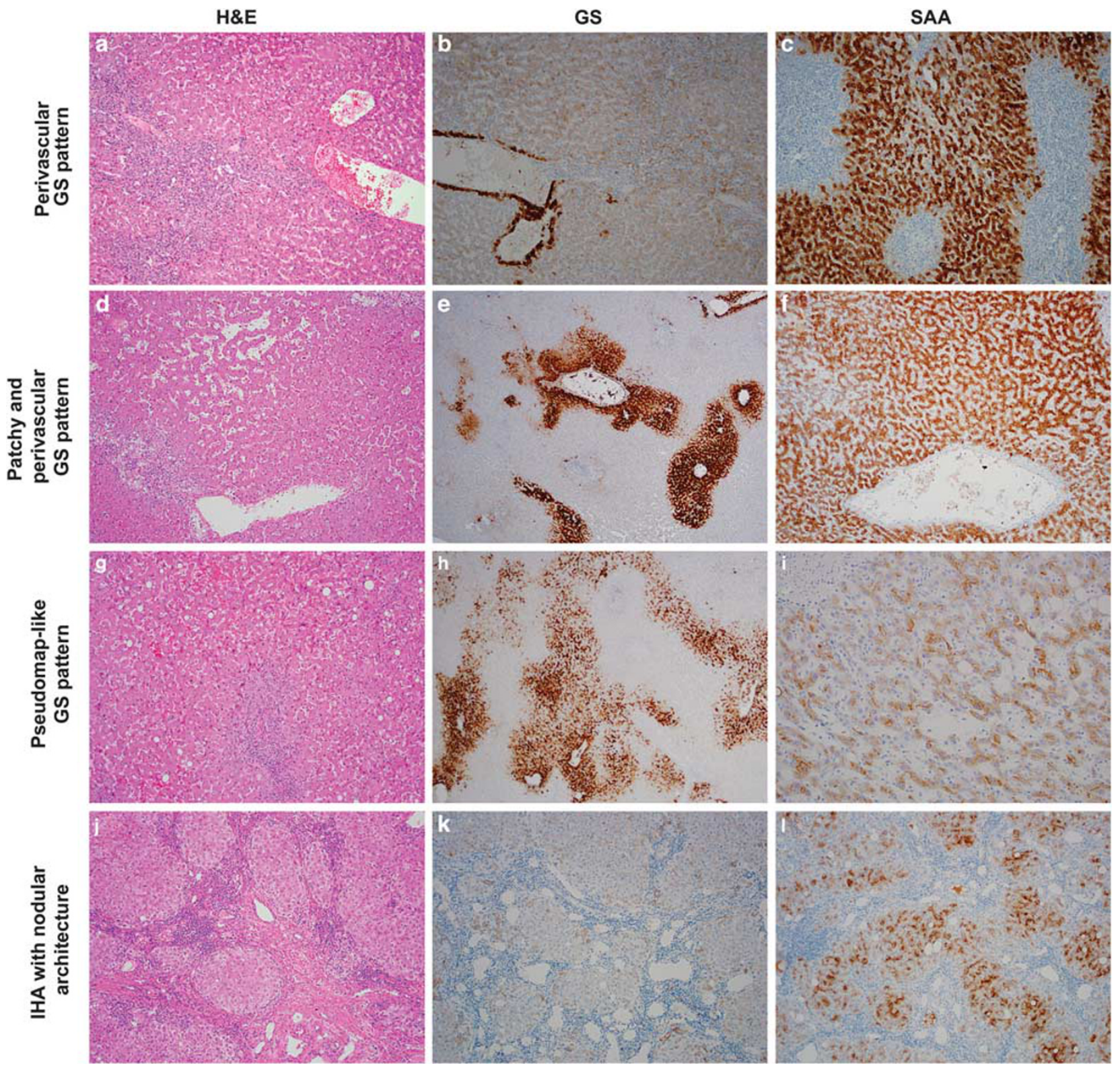

Figure 2 Glutamine synthetase and serum amyloid-associated protein staining patterns in inflammatory hepatocellular adenoma. (a-c) H\&E-stained image showing inflammation and sinusoidal dilatation (a), perivascular staining with glutamine synthetase (b), and strong cytoplasmic staining with serum amyloid-associated protein (c). (d-f) H\&E-stained image showing inflammation and sinusoidal dilatation (d), perivascular and patchy hepatocellular staining with glutamine synthetase (e), and strong cytoplasmic staining with serum amyloid-associated protein (f). (g-i) H\&E-stained image showing inflammation and sinusoidal dilatation (g), pseudo map-like staining with glutamine synthetase (h), and cytoplasmic staining with serum amyloid-associated protein (i). (j-l) H\&E-stained image with nodularity and fibrous septa resembling focal nodular hyperplasia (j), negative results with glutamine synthetase (k), and strong cytoplasmic staining with serum amyloid-associated protein (l).

hepatocellular adenoma is a benign hepatocellular neoplasm that is often surgically resected due to risk of hemorrhage and/or neoplastic transformation. ${ }^{6}$ Hence the distinction between focal nodular hyperplasia and inflammatory hepatocellular adenoma has a direct impact on patient management. Our series confirms that morphological overlap between focal nodular hyperplasia and inflammatory hepatocellular adenoma makes this distinction difficult on histological grounds.
Sinusoidal dilatation, a hallmark of inflammatory hepatocellular adenoma, was seen in $83 \%$ of cases. It is now recognized that the lesion formerly referred to as 'telangiectatic focal nodular hyperplasia' represents inflammatory hepatocellular adenoma; ;,5,7,8 however, sinusoidal dilatation was observed in $18 \%$ of true focal nodular hyperplasia cases in our series. A similar observation has been made in a recent case report. ${ }^{21}$ Similarly, naked arterioles in the parenchyma and pseudo-portal tracts have been regarded 

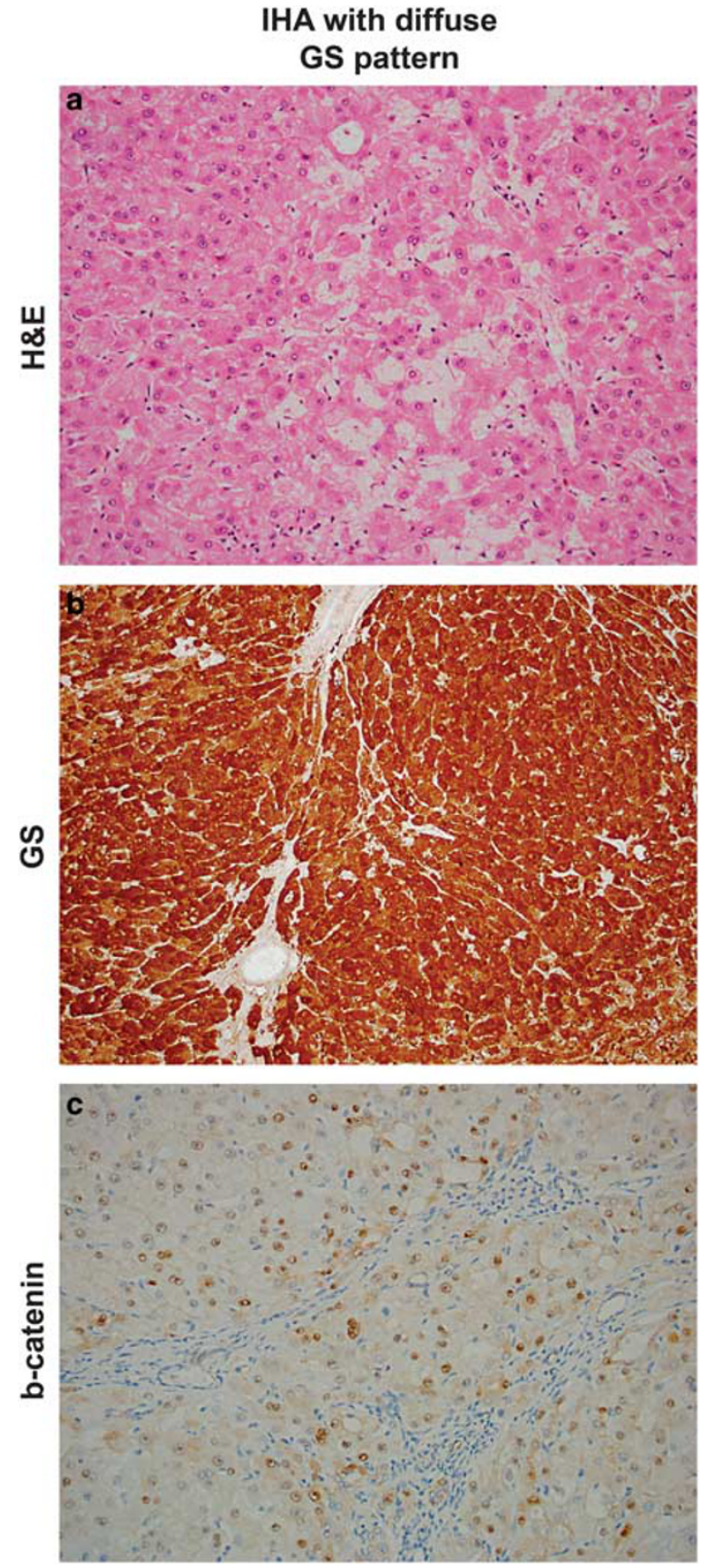

Figure 3 Inflammatory hepatocellular adenoma. (H\&E-stained image (a), with diffuse glutamine synthetase expression (b) and nuclear $\beta$-catenin expression (c)).

as characteristic of inflammatory hepatocellular adenoma, ${ }^{22}$ but both features were also observed in $22 \%$ of focal nodular hyperplasia cases in our study. Steatosis and hemorrhage were significantly more common in inflammatory hepatocellular adenoma but were observed in a minority of focal nodular hyperplasia cases; there was no significant difference in inflammation. Similar overlap of histological features has been reported in other studies. ${ }^{6}$ Nodular architecture, fibrous bands, and ductular reaction were seen in nearly all focal nodular hyperplasia cases. Inflammatory hepatocellular adenoma also showed a nodular architecture in $7 \%$ and incomplete fibrous bands in one-fourth of the cases. Ductular reaction was seen in $40 \%$ of the inflammatory hepatocellular adenoma cases, which is similar to 21 and $29 \%$ observed in other studies. ${ }^{6,23}$ In addition to the histological overlap, the lack of typical features also contributes to the difficulty in establishing the diagnosis. For example, both telangiectasia and inflammation are seen in less than half of needle biopsies of inflammatory hepatocellular adenoma. ${ }^{24}$

In view of these morphological challenges, immunohistochemical staining for glutamine synthetase and serum amyloid-associated protein is increasingly being used to confirm the diagnosis. ${ }^{14}$ Our results highlight the utility and the pitfalls in interpretation of these stains (Table 5). Glutamine synthetase shows pericentral staining in the normal liver and map-like glutamine synthetase staining in virtually all the cases of focal nodular hyperplasia. ${ }^{15,25,26}$ In our study, map-like glutamine synthetase pattern was observed in $90 \%$ of focal nodular hyperplasia, but was focal in $17 \%$ of cases, all of which were biopsies. In the remaining $10 \%$ of focal nodular hyperplasia cases, strong glutamine synthetase staining was observed in patchy groups of hepatocytes without the anastamosing map-like pattern. Hence focal or absence of map-like glutamine synthetase staining in biopsies does not exclude the diagnosis of focal nodular hyperplasia. Map-like glutamine synthetase pattern has been reported to be focal or absent in focal nodular hyperplasia cases with steatosis; ${ }^{15}$ however, we did not observe this in our study.

There is limited data in the literature on the spectrum of glutamine synthetase staining in inflammatory hepatocellular adenomas. ${ }^{15}$ In our study, most inflammatory hepatocellular adenoma cases showed perivenous glutamine synthetase staining with additional patchy areas of staining of hepatocytes in intervening region. This patchy staining shows some overlap with the $10 \%$ focal nodular hyperplasia cases with patchy glutamine synthetase staining. Unlike focal nodular hyperplasia cases where the patchy staining was uniformly intense, the patchy staining in inflammatory hepatocellular adenoma was more heterogeneous, with weak-to-moderate intensity in most hepatocytes. Similar to previous observations, the central area of the inflammatory hepatocellular adenoma did not show map-like glutamine synthetase staining in any case. ${ }^{15}$ In $15 \%$ of inflammatory hepatocellular adenomas, the patchy areas of glutamine synthetase staining at the periphery of the lesion were interconnected resembling the map-like focal nodular hyperplasia pattern. This pseudo map-like pattern was more heterogeneous and weaker in intensity compared with the classic map-pattern, and the staining decreased in intensity away from the perivascular 

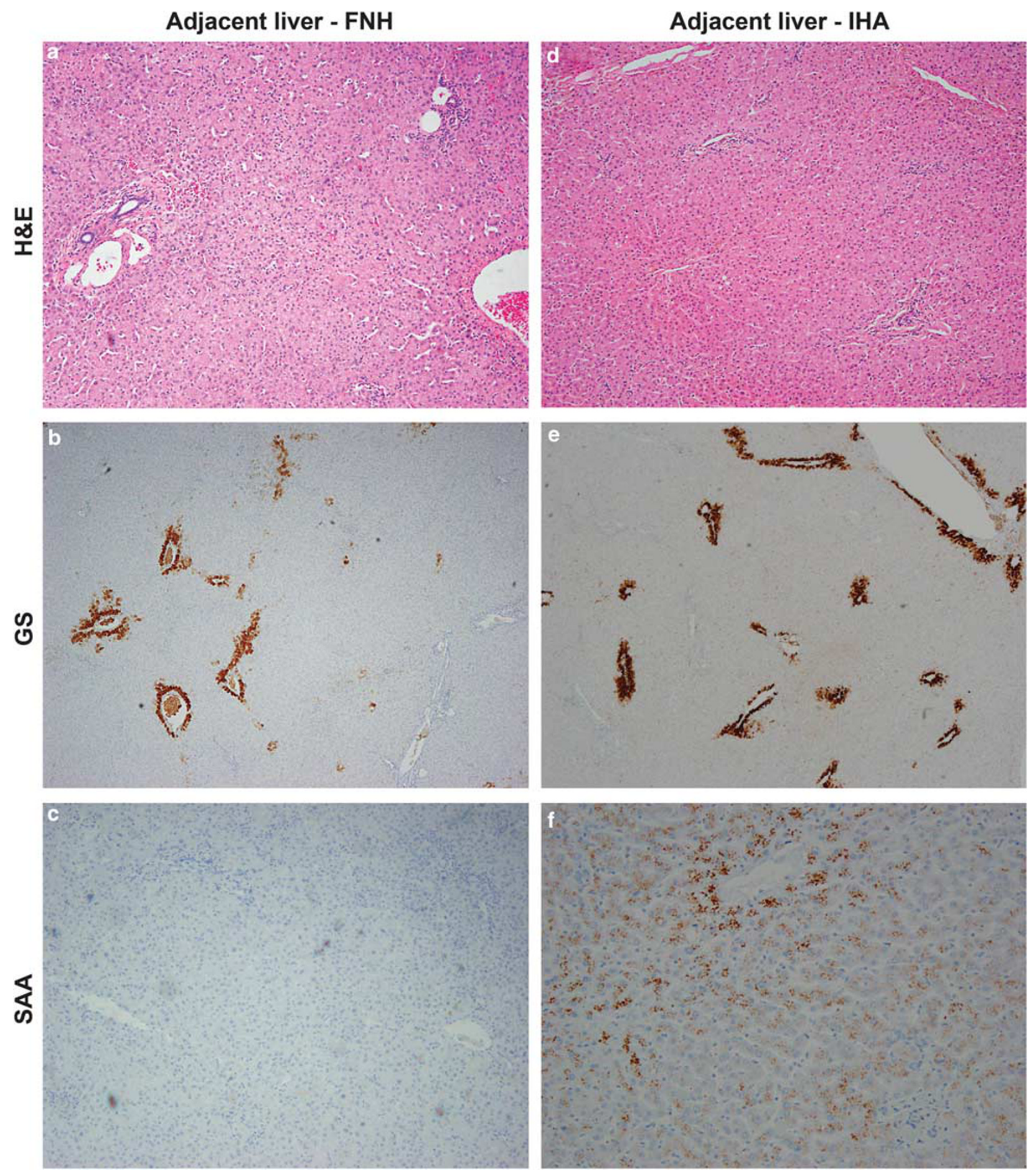

Figure 4 (a-c) Normal liver (H\&E-stained image (a), adjacent to a case of focal nodular hyperplasia, showing perivascular glutamine synthetase staining (b) and negative result with serum amyloid-associated protein staining (c)). (d-f) Normal liver (H\&E-stained image (d), adjacent to inflammatory hepatocellular adenoma case, showing perivascular glutamine synthetase staining (e) and patchy cytoplasmic staining with serum amyloid-associated protein (f)).

regions; however, the staining patterns were indistinguishable in certain areas of the lesion. Hence glutamine synthetase staining focally resembling map-like pattern should not be considered as evidence of focal nodular hyperplasia if other features support inflammatory hepatocellular adenoma. This error is especially likely to occur in the context of needle biopsies. All inflammatory hepatocellular adenomas with $\beta$-catenin activation showed diffuse glutamine synthetase staining, which was similar to earlier reported results. ${ }^{15,17}$ A combination of gp130 alterations and $\beta$-catenin 

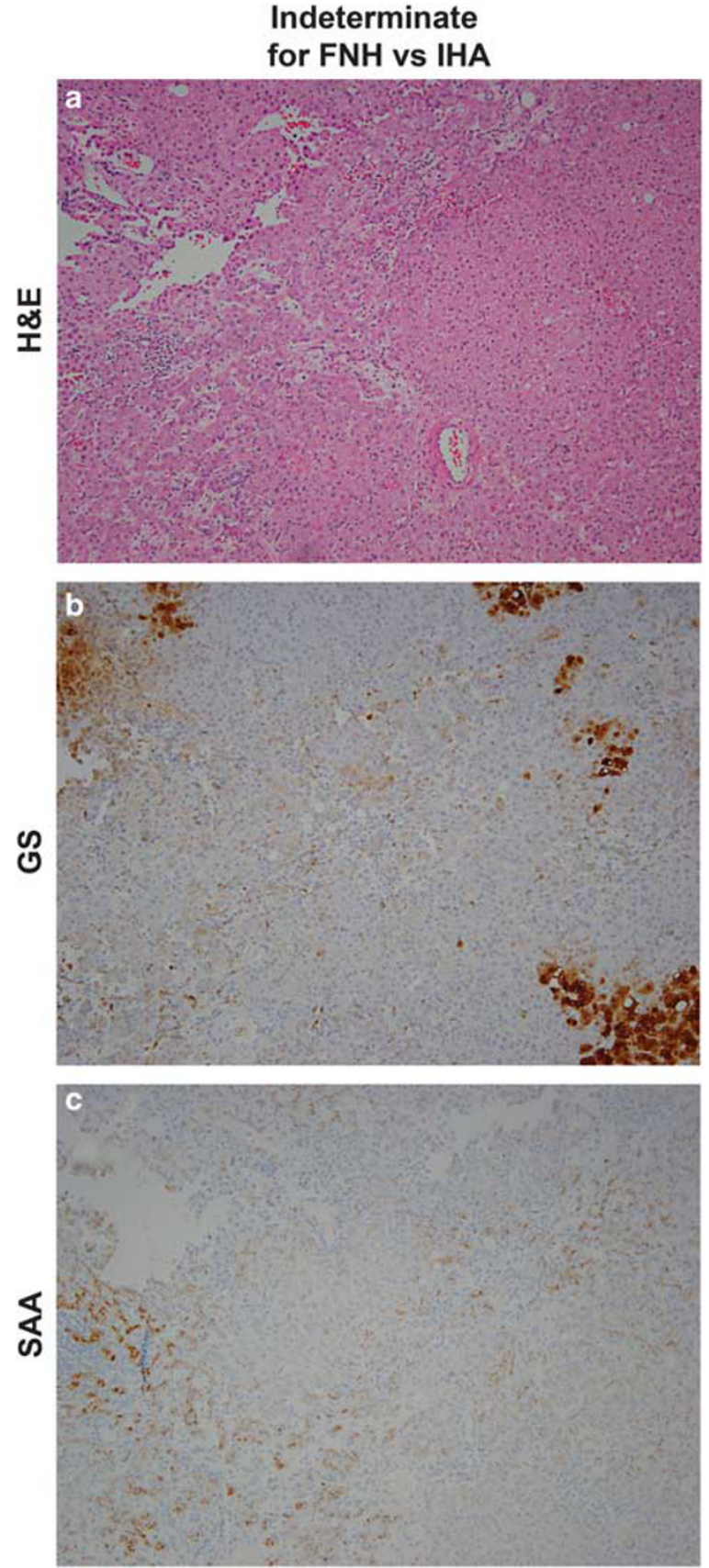

Figure 5 H\&E-stained image of an indeterminate lesion showing vague nodularity, fibrous stroma, and ductular reaction (a), with focal glutamine synthetase staining (b) and focal cytoplasmic staining with serum amyloid-associated protein (c).

mutation is likely in these cases leading to the activation of both pathways. ${ }^{20}$ The diffuse glutamine synthetase pattern in these cases is different from focal nodular hyperplasia map-like pattern, which often shows sparing of hepatocytes around the fibrous septa.

Serum amyloid-associated protein is typically not expressed in the normal liver. In our series, coarse cytoplasmic serum amyloid-associated protein staining was seen in hepatocytes in $93 \%$ of inflammatory hepatocellular adenoma, which is similar to the 90 and $94 \%$ figures reported earlier. ${ }^{17,23}$ There is limited data in the literature about serum amyloid-associated protein staining in focal nodular hyperplasia. In the series by BioulacSage $\mathrm{P}$ et al, ${ }^{17}$ all 20 focal nodular hyperplasia cases were negative for serum amyloid-associated protein, including cases that had another serum amyloidassociated protein-positive inflammatory hepatocellular adenoma in the same liver. In our series, $17 \%$ of focal nodular hyperplasia cases were serum amyloid-associated protein positive; all these cases showed the classic map-like glutamine synthetase pattern. The staining was weak to moderate and focal in majority of cases. Serum amyloid-associated protein positivity in 15\% of focal nodular hyperplasia has also been reported in a preliminary study. ${ }^{25}$

The adjacent non-lesional liver showed serum amyloid-associated protein positivity in more than half of focal nodular hyperplasia and inflammatory hepatocellular adenoma cases. Serum amyloidassociated protein staining also occurs in adjacent non-lesional liver in other primary and metastatic hepatocellular neoplasms (unpublished observations). Serum amyloid-associated protein staining has been described in non-tumor hepatocytes in $15 \%$ of inflammatory hepatic adenomas and $3 \%$ of normal liver samples. ${ }^{17}$ If a needle biopsy misses the lesion, the positive serum amyloid-associated protein along with ductular reaction and other mass-effect changes in the peritumoral region can be misinterpreted as inflammatory hepatocellular adenoma.

Our results show that C-reactive protein has a higher sensitivity than serum amyloid-associated protein and was diffusely positive in all cases of inflammatory hepatocellular adenoma. However, it has relatively low specificity with positive staining in $78 \%$ of focal nodular hyperplasia cases, with diffuse staining in $15 \%$ of cases. In view of the low specificity, C-reactive protein has limited diagnostic utility. In rare cases of inflammatory hepatocellular adenoma that are serum amyloid-associated protein negative, diffuse C-reactive protein staining along with appropriate morphology and lack of map-like glutamine synthetase pattern can be helpful to confirm the diagnosis.

The term 'focal nodular hyperplasia-like' has been used to describe lesions that morphologically resemble focal nodular hyperplasia but occur in the setting of liver disease such as cirrhosis and vascular disorders like idiopathic portal hypertension, hereditary hemorrhagic telangiectasia, and Budd-Chiari syndrome. ${ }^{27-30}$ Map-like glutamine synthetase pattern has been described in the lesions associated with vascular disorders ${ }^{29}$ but was not seen in focal nodular hyperplasia-like lesions in cirrhosis. ${ }^{16}$ In our series, three cases were indeterminate for diagnosis and showed features resembling focal nodular hyperplasia such as vague nodularity, a few incomplete fibrous bands and minimal ductular reaction with no or minimal 
Table 5 Immunohistochemical pitfalls and suggested approach in the use of glutamine synthetase (GS) and serum amyloid-associated (SAA) protein in the diagnosis of focal nodular hyperplasia (FNH) and inflammatory hepatocellular adenoma (IHA)

$\begin{array}{lll}\text { Feature Pitfall Approach } & \text { Apto }\end{array}$

SAA staining in peritumoral Misinterpreted as evidence of IHA, especially when Positive SAA is not specific for IHA. Presence of liver in needle biopsy that other peritumoral features like inflammation and missed the lesion ductular reaction are also present interlobular bile ducts and absence of diffuse CD34 staining can help in confirming that the biopsy comprises non-neoplastic liver

SAA negative in a lesion Misinterpreted as absolute evidence against IHA

SAA can be negative in $5-10 \%$ of IHA. Imaging and that shows typical features absence of map-like GS staining is needed to of IHA confirm IHA in these cases

SAA positive in a lesion that Misinterpreted as IHA shows typical features of FNH

Perivenous and patchy GS in Misinterpreted as map-like pattern of FNH a lesion that shows typical features of IHA

Diffuse GS staining Misinterpreted as map-like pattern of FNH

SAA can be seen in up to $17 \%$ of FNH. Map-like GS confirms FNH irrespective of SAA staining

This staining pattern is common in IHA. It is distinguished from map-like pattern by (i) lack of anastamosing pattern of staining and (ii) staining is heterogeneous and weak compared with homogeneous strong staining in map-like pattern

Diffuse GS staining is seen in $\beta$-catenin-activated tumors and does not have areas of periseptal sparing typical of map-like pattern. Most of these cases also show nuclear $\beta$-catenin

Pseudo map-like GS pattern Misinterpreted as map-like pattern of FNH

This pattern is seen at the periphery of IHA and $10 \%$ of FNH cases. When seen in biopsies, it is more likely to be FNH. Correlation with imaging and SAA staining is helpful. Diagnosis on needle biopsy may remain indeterminate in some cases

sinusoidal dilatation, steatosis, inflammation, or hemorrhage. As the term focal nodular hyperplasialike has been used differently in literature, we did not use this term despite the close resemblance to focal nodular hyperplasia. Similar lesions were described as 'minor' or 'subtle focal nodular hyperplasia' by Lepreux et $a l,{ }^{31}$ who proposed that these may be precursors of focal nodular hyperplasia. Our cases showed a pseudo map-like glutamine synthetase pattern similar to the staining seen at periphery of inflammatory hepatocellular adenomas; the classic map-like pattern was not present. All cases showed moderate serum amyloidassociated protein staining. All three cases were biopsy specimens, and it is possible that a definite diagnosis would have been possible on resection.

In summary, a diffuse map-like glutamine synthetase pattern confirms the diagnosis of focal nodular hyperplasia, irrespective of serum amyloidassociated protein staining. If the map-like pattern is absent or focal, other factors like intensity and heterogeneity of glutamine synthetase staining and serum amyloid-associated protein staining results have to be considered to distinguish focal nodular hyperplasia with focal map-like pattern from inflammatory hepatocellular adenoma with pseudo map-like glutamine synthetase staining at the periphery. If the glutamine synthetase is patchy and does not have map-like pattern, serum amyloidassociated protein positivity favors inflammatory hepatocellular adenoma as long as it is confirmed that the biopsy is representative of the lesion and serum amyloid-associated protein-positivity in perilesional tissue is not being overinterpreted. Finally, it is important to emphasize that glutamine synthetase and serum amyloid-associated protein immunohistochemistry should be regarded as adjuncts to diagnosis, and the final analysis must be made in light of the clinical, radiological, and morphological features.

\section{Disclosure/conflict of interest}

The authors declare no conflict of interest.

\section{References}

1 Bioulac-Sage $\mathrm{P}$, Laumonier $\mathrm{H}$, Cubel $\mathrm{G}$, et al. Over-expression of glutamine synthase in focal nodular hyperplasia (part 1): early stages in the formation support the hypothesis of a focal hyper-arterialisation with venous (portal and hepatic) and biliary damage. Comp Hepatol 2008;7:2.

2 Nguyen BN, Flejou JF, Terris B, et al. Focal nodular hyperplasia of the liver: a comprehensive pathologic study of 305 lesions and recognition of new histologic forms. Am J Surg Pathol 1999;23:1441-1454.

3 Wanless IR, Mawdsley C, Adams R. On the pathogenesis of focal nodular hyperplasia of the liver. Hepatology 1985;5:1194-1200.

4 Lepreux S, Laurent C, Le Bail B, et al. Multiple telangiectatic focal nodular hyperplasia: vascular abnormalities. Virchows Arch 2003;442:226-230.

5 Wanless IR, Albrecht S, Bilbao J, et al. Multiple focal nodular hyperplasia of the liver associated with vascular malformations of various organs and neoplasia of 
the brain: a new syndrome. Mod Pathol 1989;2: 456-462.

6 Bioulac-Sage $\mathrm{P}$, Balabaud $\mathrm{C}$, Bedossa $\mathrm{P}$, et al. Pathological diagnosis of liver cell adenoma and focal nodular hyperplasia: Bordeaux update. J Hepatol 2007;46:521-527.

7 Bioulac-Sage P, Rebouissou S, Sa Cunha A, et al. Clinical, morphologic, and molecular features defining so-called telangiectatic focal nodular hyperplasias of the liver. Gastroenterology 2005;128:1211-1218.

8 Paradis V, Benzekri A, Dargere D, et al. Telangiectatic focal nodular hyperplasia: a variant of hepatocellular adenoma. Gastroenterology 2004;126:1323-1329.

9 Paradis V, Laurent A, Flejou JF, et al. Evidence for the polyclonal nature of focal nodular hyperplasia of the liver by the study of X-chromosome inactivation. Hepatology 1997;26:891-895.

10 Paradis V, Bieche I, Dargere D, et al. A quantitative gene expression study suggests a role for angiopoietins in focal nodular hyperplasia. Gastroenterology 2003; 124:651-659.

11 Bioulac-Sage P, Balabaud C, Wanless IR. Focal nodular hyperplasia and hepatocellular adenoma, In: Bosman FT, Carneiro F, Hruban RH, Theise ND (eds). WHO Classification of Tumours of the Digestive System, 4th edn, vol. 3, Chapter 10. IARC: Lyon, France; 2010, pp 198-204.

12 Burke ZD, Tosh D. The Wnt/beta-catenin pathway: master regulator of liver zonation? Bioessays 2006;28:1072-1077.

13 Walther Z, Jain D. Molecular pathology of hepatic neoplasms: classification and clinical significance. Patholog Res Int 2011; 2011;403929.

14 Bioulac-Sage P, Cubel G, Taouji S, et al. Immunohistochemical markers on needle biopsies are helpful for the diagnosis of focal nodular hyperplasia and hepatocellular adenoma subtypes. Am J Surg Pathol 2012;36:1691-1699.

15 Bioulac-Sage P, Laumonier H, Rullier A, et al. Overexpression of glutamine synthetase in focal nodular hyperplasia: a novel easy diagnostic tool in surgical pathology. Liver Int 2009;29:459-465.

16 Rebouissou S, Couchy G, Libbrecht L, et al. The beta-catenin pathway is activated in focal nodular hyperplasia but not in cirrhotic FNH-like nodules. J Hepatol 2008;49:61-71.

17 Bioulac-Sage $\mathrm{P}$, Rebouissou S, Thomas C, et al. Hepatocellular adenoma subtype classification using molecular markers and immunohistochemistry. Hepatology 2007;46:740-748.

18 Bioulac-Sage P, Balabaud C, Zucman-Rossi J. Subtype classification of hepatocellular adenoma. Dig Surg 2010;27:39-45.
19 Pilati C, Amessou M, Bihl MP, et al. Somatic mutations activating STAT3 in human inflammatory hepatocellular adenomas. J Exp Med 2011;208:1359-1366.

20 Rebouissou S, Amessou M, Couchy G, et al. Frequent in-frame somatic deletions activate gp130 in inflammatory hepatocellular tumours. Nature 2009; 457:200-204

21 Laumonier H, Frulio N, Laurent C, et al. Focal nodular hyperplasia with major sinusoidal dilatation: a misleading entity. BMJ Case Rep 2010.

22 Suriawinata AA, Lim KH, Fiel MI, et al. Useful pathologic features to distinguish telangiectatic/ inflammatory hepatocellular adenoma from other benign hepatocellular tumors. Mod Pathol 2009;22:323A.

23 Evason KJ, Grenert JP, Ferrell LD, et al. Atypical hepatocellular adenoma-like neoplasms with betacatenin activation show cytogenetic alterations similar to well-differentiated hepatocellular carcinomas. Hum Pathol 2013;44:750-758.

24 Mounajjed T, Wu TT. Telangiectatic variant of hepatic adenoma: clinicopathologic features and correlation between liver needle biopsy and resection. Am J Surg Pathol 2011;35:1356-1363.

25 Gru A, Pai RK, Brunt EM. Clinicopathologic and molecular characterization of hepatocellular adenomas and focal nodular hyperplasia. Mod Pathol 2010;23:356A.

26 Gebhardt R, Baldysiak-Figiel A, Krugel V, et al. Hepatocellular expression of glutamine synthetase: an indicator of morphogen actions as master regulators of zonation in adult liver. Prog Histochem Cytochem 2007;41:201-266.

27 Akutsu N, Wakasugi H, Ohzeki R, et al. [Hyperplastic hepatic nodules in a case of idiopathic portal hypertension: changes in imaging findings during a 6-year follow-up]. Nihon Shokakibyo Gakkai Zasshi 2010;107:102-111.

28 Cazals-Hatem D, Vilgrain V, Genin P, et al. Arterial and portal circulation and parenchymal changes in BuddChiari syndrome: a study in 17 explanted livers. Hepatology 2003;37:510-519.

29 Cho SWI, Paradis V, et al. FNH-like lesions and glutamine synthetase expression in the liver in hereditary hemorrhagic telangiectasia. Mod Pathol 2011;24(Suppl.1):358A (abstract).

30 Libbrecht L, Cassiman D, Verslype C, et al. Clinicopathological features of focal nodular hyperplasia-like nodules in 130 cirrhotic explant livers. Am J Gastroenterol 2006;101:2341-2346.

31 Lepreux S, Laurent C, Balabaud C, et al. FNH-like nodules: possible precursor lesions in patients with focal nodular hyperplasia (FNH). Comp Hepatol 2003;2:7. 